\title{
Comparison of Metformin and Alogliptin Fixed-Dose Tablets Once a Morning Versus Once an Evening Using Continuous Glucose Monitoring (AMPM Study): An Open-Label Randomized Cross-Over Trial
}

\author{
Futoshi Ebara $^{\mathrm{a}}$, Masayuki Domichi ${ }^{\mathrm{b}}$, Akiko Suganuma $^{\mathrm{b}}$, Naoki Sakane $^{\mathrm{b}, \mathrm{c}}$
}

\begin{abstract}
Background: The aim of the study is to compare the effect of metformin hydrochloride and alogliptin benzoate combination tablets medication once daily $\mathrm{am} / \mathrm{pm}$ on blood glucose and investigate predictive factors for drug responses.
\end{abstract}

Methods: This is a single-center, single-dose, open-label, randomized, two-treatment (once-daily, am and pm), two-sequence and two-period crossover study with a washout period of 1 day. Glycemic variability and control were assessed using the FreeStyle Libre Pro continuous glucose monitoring in terms of time spent in different glycemic ranges and low/high blood glucose indices (LBGI/HBGI), and compared between the dosing timing.

Results: The average postprandial glucose in lunch and dinner in AM group were lower but not significant compared to PM group. There was no difference in average, time above range (TAR: $>180 \mathrm{mg} /$ $\mathrm{dL}$ ), time in range (TIR: $70-180 \mathrm{mg} / \mathrm{dL})$, time below range (TBR: < $70 \mathrm{mg} / \mathrm{dL}$ ), and area under curve (AUC) (AM0 - AM6, AM6 - PM0, PM0 - PM6, and PM6 - PM12) between treatments time (AM vs. PM). There was a significant strong negative correlation between highdensity lipoprotein cholesterol (HDL-C) levels and changes of HBGI from AM to PM ( $r=-0.608)$, but HDL-C levels were not associated with LBGI. There was moderately strong correlation between evening type in chronotype and changes of HBGI from AM to PM $(r=0.592)$.

Conclusions: These findings suggest that HDL-C levels and chronotype might modulate drug response, although there was no difference

Manuscript submitted December 19, 2020, accepted January 8, 2021

Published online February 3, 2021

aDepartment of the Internal Medicine, Sukoyaka Takada Central Hospital, TakaKohokutanishi, Kohoku-Ku 2-6-5, Yokohama-City, Kanagawa, Japan bDivision of Preventive Medicine, Clinical Research Institute, National Hospital Organization Kyoto Medical Center, Kyoto, Japan

${ }^{\mathrm{c} C}$ Corresponding Author: Naoki Sakane, Division of Preventive Medicine, Clinical Research Institute, National Hospital Organization Kyoto Medical Center, 1-1 Mukaihata-cho, Fukakusa, Fushimi-ku, Kyoto 612-8555, Japan. Email: nsakane@gf6.so-net.ne.jp

doi: https://doi.org/10.14740/jem720 in average, TIR, TBR, TAR, and AUC between treatments timing in patients with type 2 diabetes (T2D).

Keywords: Metformin; CGM; Chronotype; LBGI; HBGI

\section{Introduction}

Metformin is a first-line medication for patients with type 2 diabetes (T2D) [1]. On the other hand, alogliptin, which is the dipeptidyl peptidase-4 inhibitor (DPP-4i), is an oral and antidiabetic medication that is approved for patients with T2D in the world including Japan [2]. Fixed-dose combination products containing metformin and DPP-4i have been developed and improved drug adherence [3]. The blood glucose (BG) levels after breakfast were increased but BG levels after lunch were decreased after switching from twice-daily 250-mg metformin plus to once-daily 25 -mg alogliptin fixed-dose combination in patients with T2D [4]. According to the morningness-eveningness questionnaire, people were divided into morning type or evening type [5]. Flash glucose monitoring offer daily glucose profiles [6]. However, the comparative efficacy of fixed dose combination of alogliptin and metformin [7] at am vs. pm dosing regimen has not previously been determined. The aim of the study is to compare the effect of metformin hydrochloride and alogliptin benzoate fixed-dose tablets medication once-daily am/ $\mathrm{pm}$ on $\mathrm{BG}$ and investigate predictive factors for drug response.

\section{Materials and Methods}

\section{Trial design}

This study was a single-center (metformin hydrochloride 500 $\mathrm{mg}$ and alogliptin benzoate $25 \mathrm{mg}$ fixed-dose tablets), randomized, two-way crossover trial. This study consisted of two intervention periods ( 2 weeks) and washout period (1 day). Time to reach maximum plasma concentration (Tmax) of metformin is $2.5 \mathrm{~h}$, while Tmax of alogliptin is $3 \mathrm{~h}$ [8]. Patients with T2D and poor control were randomly allocated to the se- 
quence of two treatments timing: administration at am and pm.

We registered clinical trial registry (UMIN000031445). Inclusion criteria included: 1) age of 20 - 70 years, 2) T2D, and 3$) \geq 6.5 \%$ of hemoglobin A1c (HbA1c). Exclusion criteria included type 1 diabetes mellitus, glucagon-like peptide-1 (GLP-1) agonist or insulin treatment, night worker or shift worker, contraindications of metformin medication (creatinine (Cr) $\geq 1.2 \mathrm{mg} / \mathrm{dL}$ for women or $\mathrm{Cr} \geq 1.3 \mathrm{mg} / \mathrm{dL}$ for men), and history of adverse reactions to metformin or DPP-4i medication. The recruitment of the participants started on March 31, 2018 and ended in March 2019.

\section{Outcome measure}

We downloaded the raw glucose data from the FreeStyle Libre Pro continuous glucose monitoring (CGM) system (Abbott Laboratories). Glycemic variability included the following: average glucose; standard deviation (SD); high BG index (HBGI) and low BG index (LBGI) [9], time above range (TAR: $>180 \mathrm{mg} / \mathrm{dL}$ ), time in range (TIR: $70-180 \mathrm{mg} / \mathrm{dL})$, time below range (TBR: $<70 \mathrm{mg} / \mathrm{dL}$ ), area under curve (AUC), and estimated HbA1c. We obtained information on age, body mass index (BMI), and medical history. Glycemic markers (HbAlc, glycated albumin (GA), and 1, 5-anhydroglucitol (1, 5-AG), serum lipids (total cholesterol (TC), high-density lipoprotein cholesterol (HDL-C), and triglycerides (TG), total protein (TP), albumin (Alb), blood urea nitrogen (BUN), serum creatinine $\mathrm{sCr}$, urinalysis (UA), aspartate aminotransferase (AST), alanine aminotransferase (ALT), $\gamma$-glutamyl transferase (GGT), and high-sensitivity Creactive protein (hs-CRP) were measured. The chronotype was self-classified as one of three possible classes (morning type, intermediate type, and evening type) [10]. The healthy lifestyle score (HLS) was defined based on eight healthy lifestyle behaviors, namely: 1) fruit intake per day $(\geq 1)$; 2) fish intake per day ( $\geq 1)$; 3) milk consumption per day (almost daily); 4) BMI (21 $\left.-25 \mathrm{~kg} / \mathrm{m}^{2}\right)$; 5) number of hours spent in walking and/or sports ( $\geq 0.5 \mathrm{~h}$ per day and/or $\geq 5 \mathrm{~h}$ per week, respectively); 6) avoidance of smoking (nonsmokers included past smokers); 7) alcohol consumption ( $<2$ gou $=46.0 \mathrm{~g}$ ethanol $/$ day; 1 gou is a unit of Japanese liquor that contain approximately $23 \mathrm{~g}$ of ethanol); and 8) sleep duration (5.5 - 7.4h/day). We assigned 1 point for each lifestyle behavior, and totaled the points to obtain the healthy lifestyle score, which ranged from 0 to 8 [11]. Treatment satisfaction and preference was measured using 5-point Likert scale.

\section{Sample size}

Sample sizes $(n=20)$ were estimated using the previous study [12].

\section{Randomization}

The biostatistician randomly assigned patients (1:1) to either medication in the morning (A) during period 1 followed by medication in the evening during period 2 ( $\mathrm{AB}$ sequence); fur- ther, the reverse order of the medication timing (BA sequence) was also followed.

\section{Blinding}

Patients and physicians were not blinded to the medication timing. The biostatistician performed blind analysis of the final data.

\section{Statistical analysis}

Data are indicated as mean (SD). Pearson's correlation coefficient was applied to test possible significant relationships [13]. Correlation coefficient interpretation guideline was: $0.8 \leq|\mathrm{r}|$ $<1.0$ : very strong, $0.6 \leq|\mathrm{r}| \leq 0.8$ : strong, $0.4 \leq|\mathrm{r}|<0.6$ : moderately strong, $0.2 \leq|\mathrm{r}|<0.4$ : weak, $0.0<|\mathrm{r}|<0.2$ : very weak correlation. Glycemic variability was calculated using the R package gluvarpro. Using the pkcross command in Stata, treatment and carryover effect were calculated.

\section{Adverse events}

Adverse events were assessed during the intervention period, but adverse events were not obtained in the washout period.

This study protocol was approved by the Ethics Committee of Sukoyaka Takada Central Hospital (approval number: 2018-A-02); and the study was conducted in compliance with the Helsinki Declaration.

\section{Results}

Of 17 patients screened, 12 patients consented to participate in the study. One patient was drop-out due to moving. A total of 11 patients with T2D (mean age, $57.2 \pm 11.2$ years; mean BMI, $26.1 \pm 4.6 \mathrm{~kg} / \mathrm{m}^{2}$ ) were analyzed (Table 1). Medication adherence was $100 \%$. There were no differences in laboratory parameters between groups (Table 2). The average postprandial glucose levels in lunch and dinner in AM group were lower but not significant compared to PM group (Fig. 1). There were no differences in average, time above range (TAR: $>180 \mathrm{mg} / \mathrm{dL}$ ), time in range (TIR: $70-180 \mathrm{mg} / \mathrm{dL}$ ), time below range (TBR: $<70 \mathrm{mg} / \mathrm{dL}$ ), time above range (TAR: $>180 \mathrm{mg} / \mathrm{dL}$ ), and AUC (AM0 - AM6, AM6 - PM0, PM0 - PM6, and PM6 - PM12) between groups (Table 3). HDL-C levels was significantly associated with changes of HBGI from AM to PM, but not associated with LBGI (Table 4, Fig. 2). There was moderately strong correlation between evening type in chronotype and changes of HBGI from AM to PM ( $\mathrm{r}=0.592)$. The percent of patients reported high satisfaction at medication am was $18.2 \%$, although the percent of patients reported high satisfaction at pm was $0 \%$ with no difference. The number of patients reported recommended this fixed combination medication at am was $0 \%$ but at pm was $9.1 \%$ with no difference. No adverse events related with the study were observed during the intervention period. 
Table 1. Baseline Demographics and Laboratory Data of Patients With T2D

\begin{tabular}{ll}
\hline Variables & Mean (SD) \\
\hline Age, years & $57.2(11.2)$ \\
\hline BMI, kg/m² & $26.1(4.6)$ \\
HbA1c, \% & $8.2(1.5)$ \\
TC, mg/dL & $204(26)$ \\
HDL-C, mg/dL & $52(11)$ \\
TG, mg/dL & $166(97)$ \\
Chronotype, \% & 36.4 \\
\multicolumn{1}{c}{ Morning type } & 45.5 \\
\multicolumn{1}{c}{ Intermediate } & 18.2 \\
\hline Evening type & $3.8(1.7)$ \\
\hline HLS, points (out of 8$)$ &
\end{tabular}

T2D: type 2 diabetes; BMI: body mass index; HbA1c: hemoglobin A1c; TC: total cholesterol; HDL-C: high-density lipoprotein cholesterol; TG: triglycerides; HLS: healthy lifestyle score; SD: standard deviation.

\section{Discussion}

This is the first study using flash glucose monitoring to compare the effect of metformin hydrochloride and alogliptin benzoate fix-dose tablets medication once-daily am/pm on BG and investigate predictive factors for drug response. There was no difference in average BG levels between medication timing. The crossover study by DeFronzo et al indicated that there was no significant difference in mean fasting plasma glucose (FPG) values between $1,000 \mathrm{mg}$ delayed-release metformin oncedaily am and pm groups in 26 patients with T2D, although greater decreases in change of FPG were observed following once-daily am regimen [14]. On the other hand, once-daily pm dosing of DPP-4i vildagliptin decreased FPG in patients with $\mathrm{T} 2 \mathrm{D}$, although both am and pm dosings decreased postprandial glucose [15]. In this study, there was moderately strong correlation between evening type in chronotype and changes of HBGI from AM to PM $(r=0.592)$. These meanings indicates that patients with evening type were recommended at am dosing, but patients with morning type levels were recommend at pm dosing. Further examinations including large samples are required to clarify these issued in the future.

\section{HDL-C and HBGI}

HBGI are common CGM metrics used to quantify the risk of hyperglycemia. In this study, HDL-C levels were negatively correlated with changes of HBGI from AM to PM. These meanings indicate that patients with higher HDL-C levels were recommended at pm dosing, but patients with lower HDL-C levels were recommend at am dosing. However, the mechanism why HDL-C levels modulate drug response is unclear. Kashi et al showed that there was a positive association between HDL-C levels and glycemic response to metformin in a total of 150 patients with T2D [16]. Lower HDL-C levels were associated insulin resistance in women with polycystic ovary syndrome [17], but the exact mechanism of this relationship is still unclear. Metformin have antidiabetic effects through im-

Table 2. Comparison of AM or PM Administration of Drug

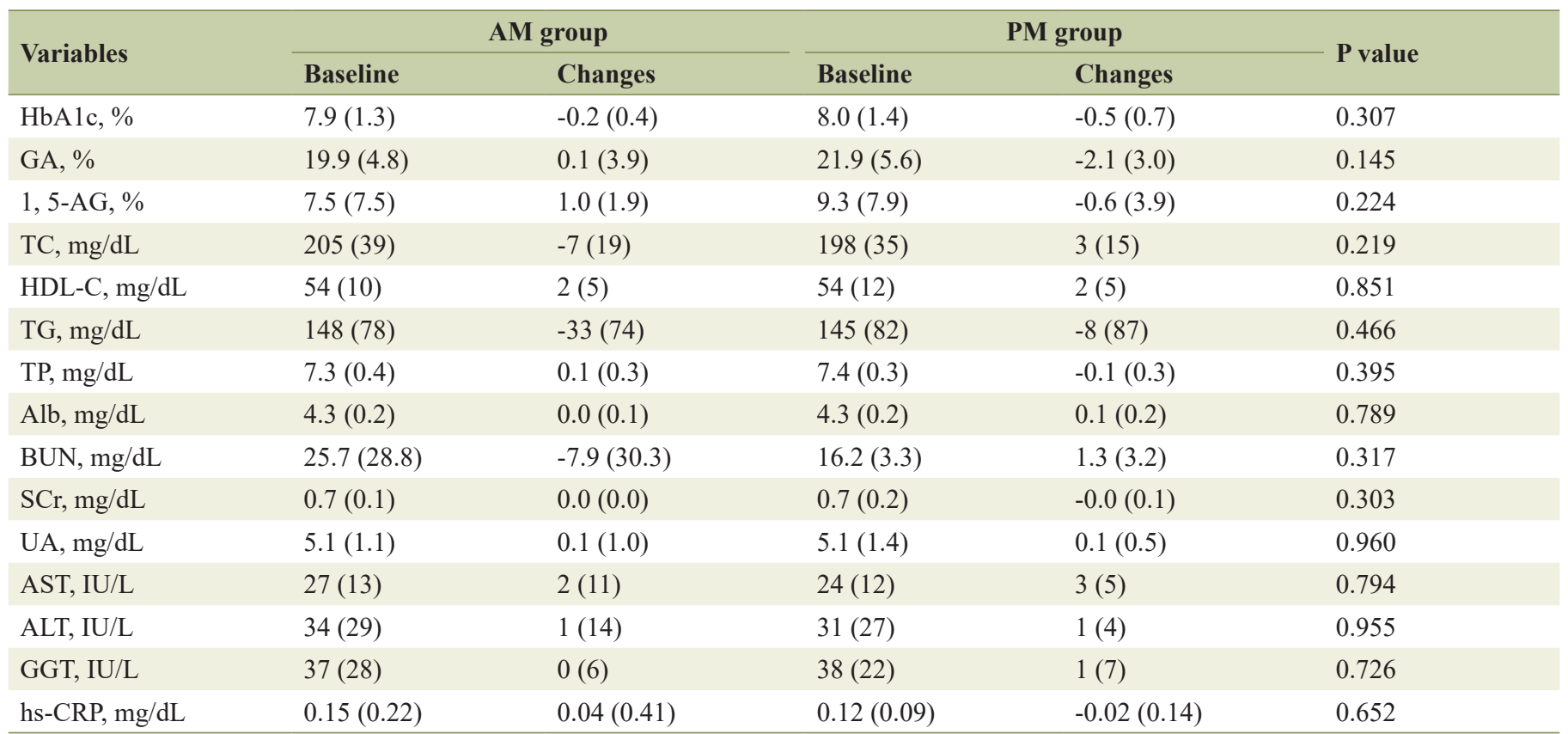

HbA1c: hemoglobin A1c; GA: glycated albumin; 1, 5-AG: 1, 5-anhydroglucitol; TC: total cholesterol; HDL-C: high-density lipoprotein cholesterol; TG: triglycerides; TC: total cholesterol; TG: triglycerides; TP: total protein; Alb: albumin; BUN: blood urea nitrogen; SCr: serum creatinine; UA: urinalysis; AST: aspartate aminotransferase; ALT: alanine aminotransferase; GGT: y-glutamyl transferase; hs-CRP: high-sensitivity C-reactive protein. 


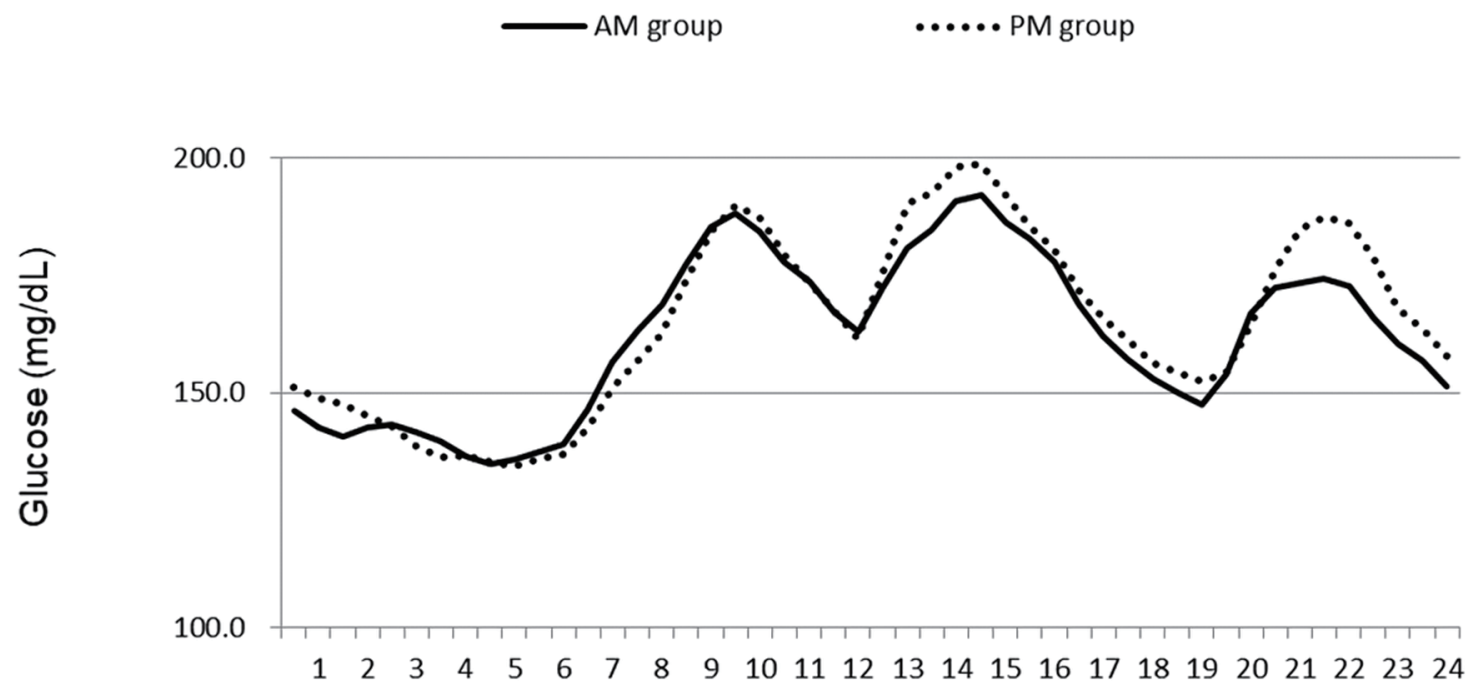

\section{Time $(\mathrm{h})$}

Figure 1. The average glucose levels in AM groups and PM groups.

proved muscular uptake of glucose and reducing hepatic gluconeogenesis, because it primarily regulated AMP-activated protein kinase (AMPK)-dependent pathways [18]. HDL might modulate glucose metabolism through activation of AMPKdependent pathways in skeletal muscle. Thus, lower HDL-C levels might affect the insulin sensitivity in the response to medication. Further examinations including insulin resistance index are required to clarify these issues.

\section{Strength and limitations}

The strengths of our study include well-designed study and high medication adherence. There were several limitations: the

Table 3. Comparison of Glucose Parameters Between Treatment Time (AM vs. PM)

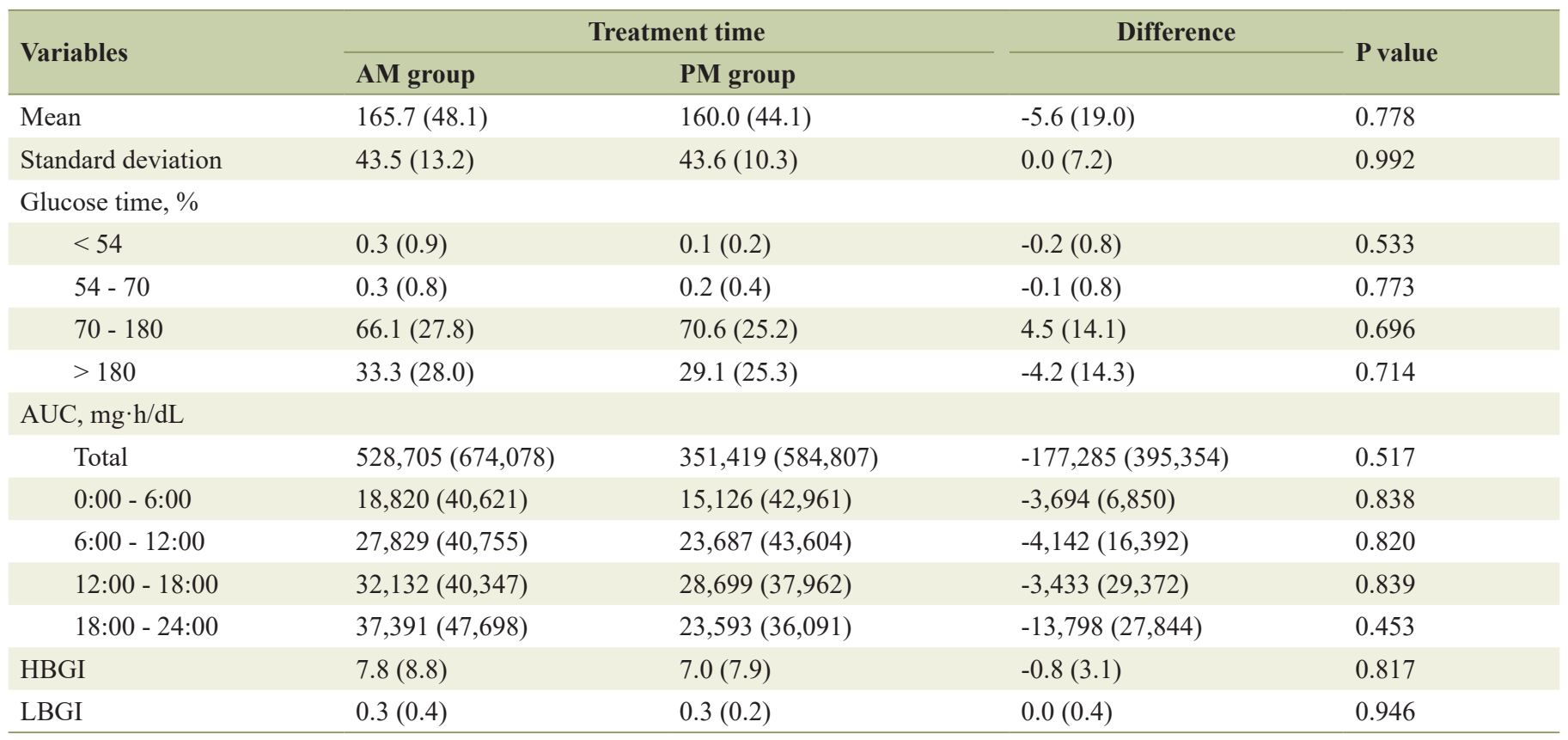

AUC: area under curve; L/HBGI: low/high blood glucose indices. 
Table 4. Correlation of Clinical Parameters With HBGI and LBGI

\begin{tabular}{|c|c|c|c|c|c|c|}
\hline \multirow{2}{*}{ Variables } & \multicolumn{3}{|c|}{ HBGI } & \multicolumn{3}{|c|}{ LBGI } \\
\hline & AM group & PM group & Difference (PM - AM) & AM group & PM group & Difference (PM - AM) \\
\hline Age & -0.284 & -0.449 & -0.347 & 0.309 & 0.121 & -0.222 \\
\hline Male & 0.253 & 0.243 & -0.099 & 0.105 & -0.454 & -0.351 \\
\hline Evening type in chronotype & -0.499 & -0.325 & 0.592 & 0.206 & 0.322 & -0.013 \\
\hline GA & 0.163 & 0.078 & -0.268 & 0.036 & 0.108 & 0.027 \\
\hline $\mathrm{TC}$ & 0.063 & 0.019 & -0.133 & -0.298 & -0.265 & 0.130 \\
\hline HDL-C & 0.261 & 0.055 & $-0.608 *$ & 0.161 & 0.003 & -0.149 \\
\hline TG & -0.095 & -0.072 & 0.088 & -0.439 & -0.136 & 0.334 \\
\hline
\end{tabular}

BMI: body mass index; HLS: healthy lifestyle score; HbA1c: hemoglobin A1c; GA: glycated albumin; TC: total cholesterol; HDL-C: high-density lipoprotein cholesterol; TG: triglycerides; L/HBGI: low/high blood glucose indices.

small sample size and short experimental period. Furthermore, it is unknown which medication affect drug response because of the alogliptin benzoate and metformin hydrochloride combination tablets. Further examinations including each study with alogliptin or metformin are required to which medication affect drug response depend on dosing timing. Therefore, these issues might have limited generalizability. Careful attention should be paid for interpreting the results, because the smaller sample size.
In conclusion, the findings of the current study suggest that chronotype and HDL-C levels might modulate drug response in patients with T2D.

\section{Acknowledgments}

We thank Dr. Chidori Ebara and Yoshinaga Akiko for collecting data and the assistance. We thank all the study investiga-

\section{$\mathrm{HDL}-\mathrm{C}$ and $\triangle \mathrm{HBGI}$}

$$
r=-0.608^{*}
$$

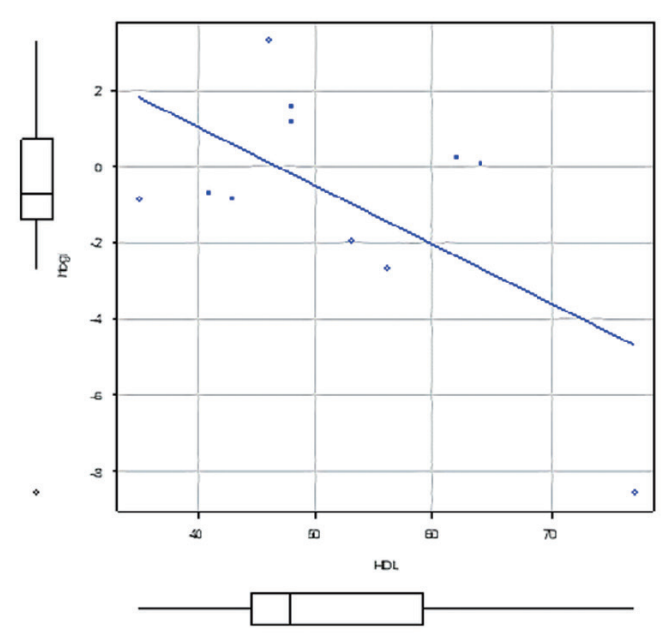

\section{$\mathrm{HDL}-\mathrm{C}$ and $\triangle \mathrm{LBGI}$}

$r=-0.149$

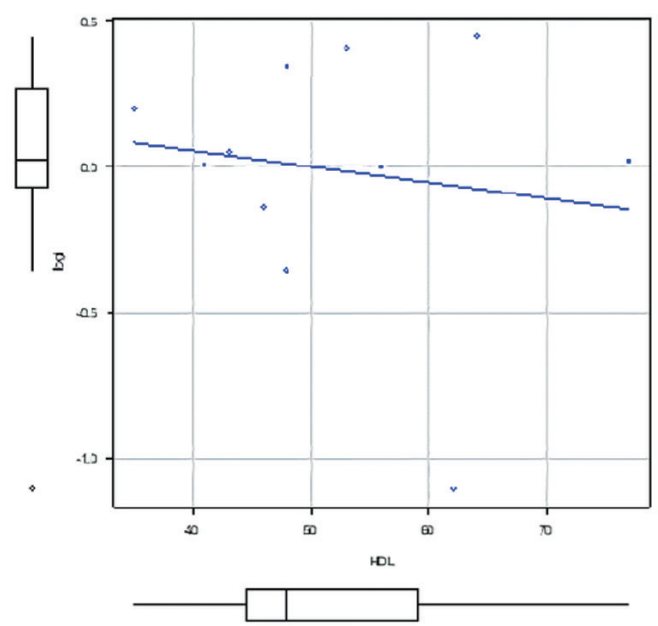

${ }^{*} P<0.05$

Figure 2. Correlation of HDL-C levels with drug response. HDL-C: high-density lipoprotein cholesterol; L/HBGI: low/high blood glucose indices. 
tors and staff and participants who participated in this study, for helpful discussions during manuscript development.

\section{Financial Disclosure}

This work was partly supported by JSPS KAKENHI (grant number: 19K02369).

\section{Conflict of Interest}

None to declare.

\section{Informed Consent}

Informed written consents were obtained from all patients.

\section{Author Contributions}

FE and NS designed and conceived the study. AS and MD analyzed the data. FE contributed to participants' data collection. FE contributed to ethical committee approval.

\section{Data Availability}

The authors declare that data supporting the findings of this study are available within the article.

\section{References}

1. Park JE, Jeong GH, Lee IK, Yoon YR, Liu KH, Gu N, Shin KH. A pharmacometabolomic approach to predict response to metformin in early-phase type 2 diabetes mellitus patients. Molecules. 2018;23(7):1579.

2. Kaku K, Kisanuki K, Shibata M, Oohira T. Benefit-risk assessment of Alogliptin for the treatment of type 2 diabetes mellitus. Drug Saf. 2019;42(11):1311-1327.

3. Neumiller JJ, Holland DQ. Alogliptin + metformin combination for the treatment of type 2 diabetes mellitus. Expert Rev Endocrinol Metab. 2016;11(1):21-31.

4. Takahara M, Shiraiwa T, Katakami N, Maeno Y, Yamamoto K, Shiraiwa Y, Yoshida Y, et al. Different daily glycemic profiles after switching from once-daily alogliptin plus twice-daily metformin to their once-daily fixed-dose combination in Japanese type 2 diabetic patients. Endocr J. 2019;66(1):11-17.

5. Brady EM, Hall AP, Baldry E, Chatterjee S, Daniels LJ, Edwardson C, Khunti K, et al. Rationale and design of a cross-sectional study to investigate and describe the chronotype of patients with type 2 diabetes and the effect on glycaemic control: the CODEC study. BMJ Open. 2019;9(11):e027773.

6. Kudva YC, Ahmann AJ, Bergenstal RM, Gavin JR, 3rd, Kruger DF, Midyett LK, Miller E, et al. Approach to Using Trend Arrows in the FreeStyle Libre Flash Glucose Monitoring Systems in Adults. J Endocr Soc. 2018;2(12):1320-1337.

7. Zaghary WA, Mowaka S, Hassan MA, Ayoub BM. Comparative study between different simple methods manipulating ratio spectra for the analysis of alogliptin and metformin co-formulated with highly different concentrations. Spectrochim Acta A Mol Biomol Spectrosc. 2017;186:23-28.

8. Christopher R, Covington P, Davenport M, Fleck P, Mekki QA, Wann ER, Karim A. Pharmacokinetics, pharmacodynamics, and tolerability of single increasing doses of the dipeptidyl peptidase- 4 inhibitor alogliptin in healthy male subjects. Clin Ther. 2008;30(3):513-527.

9. Kovatchev BP, Cox DJ, Gonder-Frederick LA, Clarke W. Symmetrization of the blood glucose measurement scale and its applications. Diabetes Care. 1997;20(11):16551658.

10. Turco M, Corrias M, Chiaromanni F, Bano M, Salamanca M, Caccin L, Merkel C, et al. The self-morningness/ eveningness (Self-ME): An extremely concise and totally subjective assessment of diurnal preference. Chronobiol Int. 2015;32(9):1192-1200.

11. Eguchi E, Iso H, Tanabe N, Wada Y, Yatsuya H, Kikuchi $\mathrm{S}$, Inaba $\mathrm{Y}$, et al. Healthy lifestyle behaviours and cardiovascular mortality among Japanese men and women: the Japan collaborative cohort study. Eur Heart J. 2012;33(4):467-477.

12. Sakamoto M, Nishimura R, Irako T, Tsujino D, Ando K, Utsunomiya K. Comparison of vildagliptin twice daily vs. sitagliptin once daily using continuous glucose monitoring (CGM): crossover pilot study (J-VICTORIA study). Cardiovasc Diabetol. 2012;11:92.

13. Akoglu H. User's guide to correlation coefficients. Turk J Emerg Med. 2018;18(3):91-93.

14. DeFronzo RA, Buse JB, Kim T, Burns C, Skare S, Baron A, Fineman M. Once-daily delayed-release metformin lowers plasma glucose and enhances fasting and postprandial GLP-1 and PYY: results from two randomised trials. Diabetologia. 2016;59(8):1645-1654.

15. He YL, Valencia J, Zhang Y, Schwartz SL, Ligueros-Saylan M, Foley J, Dole WP. Hormonal and metabolic effects of morning or evening dosing of the dipeptidyl peptidase IV inhibitor vildagliptin in patients with type 2 diabetes. Br J Clin Pharmacol. 2010;70(1):34-42.

16. Kashi Z, Mahrooz A, Kianmehr A, Alizadeh A. The role of metformin response in lipid metabolism in patients with recent-onset type 2 diabetes: HbAlc level as a criterion for designating patients as responders or nonresponders to metformin. PLoS One. 2016;11(3):e0151543.

17. Maciel GA, Hayashida SA, da Costa LC, Marcondes JA, da Fonseca AM, Soares JM, Jr., Baracat EC. Influence of LH and high-density lipoprotein cholesterol (HDLC) on metformin response in women with polycystic ovary syndrome. Eur J Obstet Gynecol Reprod Biol. 2011;157(2):180-184.

18. Chen S, Zhou J, Xi M, Jia Y, Wong Y, Zhao J, Ding L, et al. Pharmacogenetic variation and metformin response. Curr Drug Metab. 2013;14(10):1070-1082. 